\section{Contamination of disposable tonometer prisms during tonometry}

\begin{abstract}
Aim Due to the theoretical possibility of prion transmission in applanation tonometry, many ophthalmological units in the United Kingdom now use disposable tonometer prisms. We have investigated the potential for bacterial and viral transmission from the health practitioner to the patient via disposable prisms.

Methods All staff who perform applanation tonometry at the Sussex Eye Hospital (SEH) received a questionnaire to evaluate if the applanating face of the prism is touched during tonometry and the ease of use of the disposable prism compared to the reusable prisms that were previously used. We then cultured prisms handled by a random sample of staff members for common bacteria. Finally, we constructed a model to investigate the possibility of interpatient adenoviral transmission via disposable tonometer prisms. Results The questionnaire revealed that almost $50 \%$ of the staff admit to touching the applanating face of the tonometer prism prior to applanation. Cultures of the prisms grew a range of bacteria including Staphylococcus epidermidis, Staphylococcus aureus, and Bacillus species. The viral model suggested that adenovirus could be transmitted by applanation tonometry.

Conclusion The use of disposable prisms for applanation tonometry may reduce the risk of prion transmission but is not bacteriologically or virologically aseptic. This is a potential infection risk to patients.

Eye (2006) 20, 358-361. doi:10.1038/sj.eye.6701866; published online 15 April 2005
\end{abstract}

The authors have no proprietary interests.

There has been no funding for this piece of research.

This research has been presented as a poster at the Oxford Ophthalmological Congress 2004 (poster number 58)
Keywords: tonometry; infection; disposable; prism

\section{Introduction}

The use of reusable tonometer prisms for applanation tonometry has been proposed as
SN Rajak, J Paul, V Sharma and S Vickers

a source of infection of Creutzfeldt-Jakob disease (CJD). ${ }^{1}$ In view of this many ophthalmology units in the UK use disposable tonometer prisms. The Sussex Eye Hospital (SEH) started using the Tonosafe disposable tonometer prisms (Clement Clarke International) in 2002. This system consists of a small transparent plastic prism fitted onto a larger black plastic body, which in turn fits into the tonometry unit (Figure 1). While setting up and then using the system the applanating face of the prism should not be touched in order to maintain sterility. We were concerned that the applanating face was regularly being touched by many of those performing tonometry at the SEH, thereby contaminating it prior to touching the corneal surface of the patient. We investigated this concern in three parts. Firstly, we constructed a questionnaire to assess how easy or difficult members of the staff of the SEH find the disposable prism to use and whether they touch the face of the tonometer prism while performing applanation tonometry. Secondly, we assessed whether the prisms become bacterially contaminated by contact by culturing 25 tonometer prisms that had had their applanating faces deliberately touched by 25 randomly selected hospital staff. Thirdly, we constructed a laboratory virus transmission model to investigate the possibility of adenovirus transmission between patients by tonometry.

Materials and methods

Questionnaire investigating current practice of staff who perform Goldman applanation tonometry at the SEH

In all, 41 people in the SEH perform Goldman applanation tonometry regularly. They were each sent an anonymous questionnaire asking the following questions:

(1) Do you ever touch the clear plastic disposable head of the tonometer unit? 


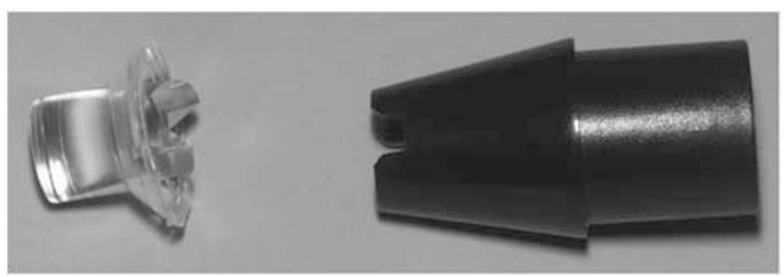

Figure 1 Disposable tonometer prism and head.

(2) If yes, do you ever touch the flat applanating face of the disposable head?

(3) If yes, do you touch it always, regularly or occasionally?

(4) How easy do you find the disposable tonometer heads to use on a scale of 1 (very) to 5 (not at all)?

(5) How easy do you find the reusable tonometer heads to use on a scale of 1 (very) to 5 (not at all)?

\section{Culture of disposable tonometer prisms contaminated by staff who perform Goldman applanation tonometry at the SEH}

In all, 25 of these staff members were selected at random. They were approached during routine ophthalmological clinic and were asked to remove a disposable tonometer prism from its blister pack and rub it between thumb and forefinger for 2-3s ensuring they do touch the applanating face. They were asked not to wash their hands prior to performing this exercise. The tonometer prism was dropped by the staff member in a sterile container and sent to the laboratory, where it was cultured for bacteria by both direct contact (of the applanating face of the prism) and enrichment methods (of the whole prism).

\section{Adenovirus transmission model}

Two Schirmers strips were inserted into the lower lids of one of the authors (SR) without topical anaesthetic in order to saturate them in tears. Each saturated strip was placed in a sterile container. In the laboratory one of the strips was inoculated with high titre adenovirus culture. This strip was held with a gloved hand to mimic a doctor holding the lid of a patient with adenoviral conjunctivitis. The gloved hand was then used to handle a previously sterile disposable tonometer prism. This was placed on a further uninoculated Schirmers strip to mimic applanation tonometry of a second patient by the same clinician. At each stage of the process samples were cultured to assess for the presence of adenovirus. The above process was performed twice.

\section{Results \\ Questionnaire}

In all, 35 of the 41 questionnaires were returned. From these it was determined that 16 of the 35 people admit to touch the applanating face of the tonometer tip when setting up the unit. Of these 16 people, five touch the applanating face regularly, and 11 occasionally.

Additionally, from the questionnaires it was established that on a scale of 1-5 (1 represents 'very easy' and 5 is 'difficult') the average score for the 35 staff members filling in this question, for use of the disposable prisms was 3.2. The average score of the 34 staff members who answered this question (one member had never used the reusable prisms) for the use of the reusable tonometer prisms 1.7 .

\section{Culture of tonometer prisms}

In all, 25 tonometer prisms that had been touched by a random selection of the tonometry performing staff of the SEH, were cultured in the laboratory. A total of 11 of these grew coagulase negative Staphylococcus epidermidis (Figure 2) (eight of these were positive to both direct culture of the prism face and by enrichment method of the whole prism, two were only positive via the enrichment method and, one was positive by direct culture only). Two of them grew Bacillus species (enrichment only). One grew both Staphylococcus aureus and coagulase negative $S$. epidermidis (both bacteria grown with direct culture and enrichment method). One grew micrococcus species (direct culture only). In all, 10 of these had no growth.

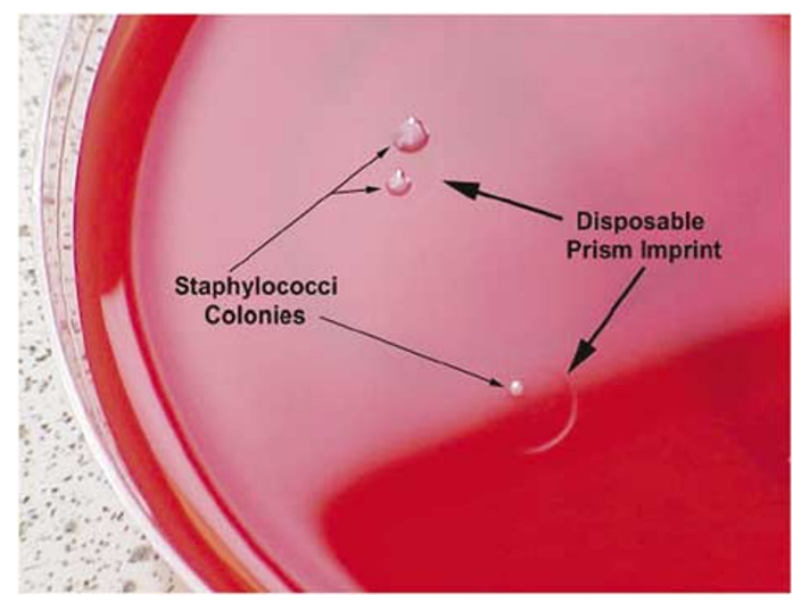

Figure 2 Direct culture of coagulase negative S. aureus from the applanating face of a disposable tonometer prism. 


\section{Adneovirus transmission model}

The experiment was performed twice, in identical fashion. Both times the adenovirus could be cultured from the initial inoculated, tear-saturated Shirmer's strips, from the gloved hand that handled the Shirmer's strips and from the tonometer tips that was then held by this hand. The final tear-saturated Shirmer's strip that was touched by the now-inoculated tonometer tip did not grow adenovirus in either experiment.

\section{Discussion}

The questionnaire findings suggest that there is significant variability in the way that the Tonosafe tonometer prism is being used at the SEH. Almost half of those using the disposable prism admit to touching the applanating face (16 out of 35 ). Five people admit to doing this regularly and 11 people occasionally. Each time this occurs it represents a breach of aseptic practice. This contamination occurs immediately prior to contact with the patient's cornea. This may occur as people find this system difficult to use (average score 3.2 out of 5 for difficulty). We did not formally investigate whether people touched the applanating face of the reusable tonometer prisms that were previously used in the eye hospital. However, personal discussion with almost all those answering the questionnaire found that the size and nature of the reusable unit made it highly improbable that the applanating face would be touched. It is a $30 \mathrm{~mm}$ long unit that can easily be held between thumb and forefinger while inserting in the tonometer head socket. This is further supported by the findings on how easy people found the non disposable units to use. The majority (20 out of 34) answered that they were very easy ( 1 out of 5 ) to use. A further eight report gave a score of 2 out of 5 . Only four staff members gave a score of 4 or 5 out of 5 . These results gave an average of 1.7 .

The culture of prisms held by 25 randomly selected staff members from the 35 that filled in the questionnaire, confirm that the practice of touching the applanating face of the tonometer prisms during use does place a detectable bacterial load on the face of the tonometer prism. This face comes into contact with the patient's cornea. In doing this part of the study the staff member was deliberately asked not to wash their hands. While they may wash their hands regularly between examining patients, it was assumed that they are likely to have shaken hands with the patient, helped them into an examination chair, held open the eyelids for ophthalmic examination and handled the tonomter unit prior to inserting the disposable head. All of these recontaminate recently washed hands. It was therefore a more realistic simulation of actual practice to approach the staff member in their clinic and ask them to handle the prism then and there without washing their hands prior to performing this exercise. Several different bacteria were cultured from the tonometer prisms. S. aureus was cultured from one prism. S. epidermidis was cultured from 11 prisms. Both of these staphylococci have been shown to cause an array of ophthalmological pathology including conjunctivitis and endophthalmitis. ${ }^{2}$ It is likely that the Bacillus species and micrococcus are air contaminants and are not potential sources of pathological infection. A healthy tear film covering an intact healthy eye provides good protection against bacteria. However, it can be postulated that if any of these defences is breached, such as a poor tear film, or immunocompromised patient, or wounded/recently operated eye, then patients are put at risk by this breach of apsesis.

Adenovirus can cause acute follicular conjunctivitis and epidemic keratoconjunctivitis. ${ }^{3}$ Epidemic conjunctivitis is highly contagious and causes significant morbidity. It spreads easily between otherwise healthy people such. Epidemics have been shown to originate from eye units or other medical facilities. ${ }^{4}$ Several papers have suggested that tonometry is the source of eye hospital initiated outbreaks. ${ }^{5,6}$ However, it has also been shown that adenovirus contamination via reusable prisms can be prevented with adequate sterilization. ${ }^{7}$ An untouched disposable tonometer prism is obviously not a source of adenovirus infection. However, we constructed the adenoviral transmission model in order to determine if a prism that has been contaminated by a staff member touching its face, could be a source of transmission. The model suggested that adenovirus could be transferred from tears to a health practitioners hands and to a disposable tonometer tip. This implies it could go from the tears of one patient to a tonometer tip that is about to used on another patient via a doctor's hands. Although adenovirus was not cultured on the final tear sample, it is clear that the second patient is at risk. Therefore, disposable tonometer prisms could be the source of a hospital-based adenovirus outbreak.

In all, 19 of the 35 people who filled in the questionnaire, do not admit to touching the applanating face of the prism during tonometry. It can be presumed that they use a good technique. As the questionnaire was anonymous we could not identify who they were, but personal communication with many participants suggested, the best technique, to be the following. (1) Expose the disposable prism in its blister packet but do not touch it or remove it from its blister, (2) hold the plastic head between thumb and forefinger with the protrusions of the prism lined up with the indents of the head, (3) press the head firmly onto the prism, 
and (4) ensure the prism is firmly embedded in the head and mount on the tonometer unit.

In conclusion, the questionnaire and subsequent culture of the tonometer prisms display a concerning breach of aseptic practice and the virus trasmission model suggests that tonometry using disposable prisms is a potential source of adenovirus infection.

\section{References}

1 Amin SZ, Smith L, Luthbert P J, Cheetham ME, Buckley RJ Minimising the risk of prion transmission by contact tonometry. Br J Ophthalmol 2003; 87: 1360-1362.
2 Mandell GL, Douglas RG, Bennett JE. Principles and Practice of Infectious Diseases, 5th ed. Churchill Livingstone: New York, 2000.

3 Fields BN, Knipe DM, Howley PM (eds). Fields' Virology, 3rd edn. Lippincott-Raven: Philadelphia, 1996.

4 Dawson CR, Hanna L, Wood TR, Despain R. Adenovirus type 8 keratoconjunctivitis in the United States. Am J Ophthalmol 1970; 69: 473-480.

5 Mueller AJ, Klauss V. Main sources of infection in 145 cases of epidemic keratoconjunctivitis. German J Ophthalmol 1993; 2: 224-227.

6 Colon LE. Keratoconjunctivitis due to adenovirus type 8: report on a large outbreak. Ann Ophthalmol 1991; 23: 63-65.

7 Craven ER, Butler SL, McCulley JP, Luby JP. Applanation tonometer tip sterilization for adenovirus type 8 . Ophthalmology 1987; 94: 1538-1540. 